Research Article

\title{
Fuzzy Fractional Evolution Equations and Fuzzy Solution Operators
}

\author{
Atimad Harir (D, Said Melliani (D), and Lalla Saadia Chadli \\ Laboratory of Applied Mathematics and Scientific Computing, Sultan Moulay Slimane University, P.O. Box 523, \\ Beni Mellal 23000, Morocco \\ Correspondence should be addressed to Atimad Harir; atimad.harir@gmail.com
}

Received 25 April 2019; Revised 5 August 2019; Accepted 31 August 2019; Published 14 November 2019

Academic Editor: Jose A. Sanz

Copyright (C) 2019 Atimad Harir et al. This is an open access article distributed under the Creative Commons Attribution License, which permits unrestricted use, distribution, and reproduction in any medium, provided the original work is properly cited.

In this paper, the fuzzy fractional evolution equations of order $q$ (FFEE) with fuzzy Caputo fractional derivative are introduced. We study the existence and uniqueness of mild solutions for FFEE under some conditions. Also, we generalize the definition of the fuzzy fractional integral and derivative order $q$. The fuzzy Laplace transform is presented and proved. The solvability of the problem (FFEE) and the properties of the fuzzy solution operator and its generator are investigated and developed.

\section{Introduction}

The fuzzy fractional differential equations (FFDEs) can also offer a more comprehensive account of the process or phenomenon. This has recently captured much attention in FFDEs. As the derivative of a function is defined in the sense of Riemann-Liouville, Grünwald-Letnikov, or Caputo in fractional calculus, the used derivative is to be specified and defined in FFDEs as well. FFDEs are examined in [1-5]. We adopted the fuzzy Laplace transform method to solve FFDEs because it has the advantage that it solves problems directly without determining a general solution and obtaining nonhomogeneous differential equations [5].

C. G. Gal and S. G. Gal studied [6], with more details, fuzzy linear and semilinear (additive and positive homogeneous) operator theory on the complete metric space.

Let $q \in \mathbb{R}_{+}, a>0$, and $\mathbb{R}_{\mathscr{F}}$ be the set of fuzzy real numbers. Our aim in this paper is to investigate the existence and uniqueness of the fuzzy mild solution for the fuzzy fractional evolution equation:

$$
\begin{cases}D^{q} \mathcal{u}(t)=A u(t)+f(t, u(t)), & t \in T=[0, a], \\ \left.D^{j} u(t)\right|_{t=0}=u_{j}(0), & j=0,1,2, \ldots, k=[q],\end{cases}
$$

where $A: D(A) \subset \mathbb{R}_{\mathscr{F}} \longrightarrow \mathbb{R}_{\mathscr{F}}$ is the infinitesimal generator of a $q$-resolvent family $\left(S_{q}(t)\right)_{t \geq 0}$, defined as $\mathbb{R}_{\mathscr{F}}, f \in T \times$
$\mathbb{R}_{\mathscr{F}} \longrightarrow \mathbb{R}_{\mathscr{F}}$ satisfies some conditions that will be specified later, and the fuzzy fractional derivative $D^{q}$ is understood here in the caputo sense.

\section{Preliminaries}

In this section, we introduce notations, definitions, and preliminary facts which are used throughout this paper.

Let us denote by $\mathbb{R}_{\mathscr{F}}=\{u: \mathbb{R} \longrightarrow[0,1]\}$ the class of fuzzy subsets of the real axis satisfying the following properties [7]:

(i) $u$ is normal, i.e., there exists an $x_{0} \in \mathbb{R}$, such that $u\left(x_{0}\right)=1$.

(ii) $u$ is fuzzy convex, i.e., for $x, y \in \mathbb{R}$ and $0<\lambda \leq 1$,

$$
u(\lambda x+(1-\lambda) y) \geq \min [u(x), u(y)] .
$$

(iii) $u$ is upper semicontinuous.

(iv) $[u]^{0}=\operatorname{cl}\{x \in \mathbb{R} \mid u(x)>0\}$ is compact.

Then, $\mathbb{R}_{\mathscr{F}}$ is called the space of fuzzy numbers. Obviously, $\mathbb{R} \subset E$. For $0<\alpha \leq 1$, denote $[u]^{\alpha}=\{x \in \mathbb{R} \mid u(x) \geq \alpha\}$, and then from (i) to (iv), it follows that the $\alpha$-level set $[u]^{\alpha} \in P_{K}(\mathbb{R})$ for all $0 \leq \alpha \leq 1$ is a closed bounded interval which we denote by $[u]^{\alpha}=\left[u_{1}^{\alpha}, u_{2}^{\alpha}\right]$. 
Where $P_{K}(\mathbb{R})$ denotes the family of all nonempty compact convex subsets of $\mathbb{R}$ and defines the addition and scalar multiplication in $P_{K}(\mathbb{R})$ as usual. For later purposes, we define $\widehat{0} \in \mathbb{R}_{\mathscr{F}}$ as

$$
\widehat{0}(t)= \begin{cases}1, & t=0 \\ 0, & t \neq 0\end{cases}
$$

Theorem 1 (see [8]). If $u \in \mathbb{R}_{\mathscr{F}}$, then

(i) $[u]^{\alpha} \in P_{K}(\mathbb{R})$ for all $0 \leq \alpha \leq 1$.

(ii) $[u]^{\alpha_{2}} \subset[u]^{\alpha_{1}}$ for all $0 \leq \alpha_{1} \leq \alpha_{2} \leq 1$.

(iii) $\left\{\alpha_{k}\right\} \subset[0,1]$ is a nondecreasing sequence which converges to $\alpha$ and then,

$$
[u]^{\alpha}=\bigcap_{k \geq 1}[u]^{\alpha_{k}} .
$$

Conversely, if $A_{\alpha}=\left\{\left[u_{1}^{\alpha}, u_{2}^{\alpha}\right] ; \alpha \in(0,1]\right\}$ is a family of closed real intervals verifying (i) and (ii), then $\left\{A_{\alpha}\right\}$ defined a fuzzy number $u \in \mathbb{R}_{\mathscr{F}}$ such that $[u]^{\alpha}=A_{\alpha}$.

Lemma 1 (see [9]). Let $u, v: X \longrightarrow[0,1]$ be the fuzzy sets.

Then, $u=v$ if and only if $[u]^{\alpha}=[v]^{\alpha}$ for all $\alpha \in[0,1]$

The following arithmetic operations on fuzzy numbers are well known and frequently used below. If $u, v \in \mathbb{R}_{\mathscr{F}}$, then

$$
\begin{aligned}
& {[u+v]^{\alpha}=\left[u_{1}^{\alpha}+v_{1}^{\alpha}, u_{2}^{\alpha}+v_{2}^{\alpha}\right],} \\
& {[u-v]^{\alpha}=\left[u_{1}^{\alpha}-v_{2}^{\alpha}, u_{2}^{\alpha}-v_{1}^{\alpha}\right],} \\
& {[\lambda u]^{\alpha}=\lambda[u]^{\alpha}= \begin{cases}{\left[\lambda u_{1}^{\alpha}, \lambda u_{2}^{\alpha}\right],} & \text { if } \lambda \geq 0, \\
{\left[\lambda u_{2}^{\alpha}, \lambda u_{1}^{\alpha}\right],} & \text { if } \lambda<0 .\end{cases} }
\end{aligned}
$$

For $u, v \in \mathbb{R}_{\mathscr{F}}$, if there exists $w \in \mathbb{R}_{\mathscr{F}}$ such that $u=v+w$, then $w$ is the Hukuhara difference of $u$ and $v$ denoted by $u-{ }^{h} v$.

Define $d: \mathbb{R}_{\mathscr{F}} \times \mathbb{R}_{\mathscr{F}} \longrightarrow \mathbb{R}^{+} \cup\{0\}$ by the following equation:

$$
d(u, v)=\sup _{\alpha \in[0,1]} d_{H}\left([u]^{\alpha},[v]^{\alpha}\right), \quad \text { for all } u, v \in \mathbb{R}_{\mathscr{F}},
$$

where $d_{H}$ is the Hausdorff metric defined in $P_{K}(\mathbb{R})$.

$$
d_{H}\left([u]^{\alpha},[v]^{\alpha}\right)=\max \left\{\left|u_{1}^{\alpha}-v_{1}^{\alpha}\right|,\left|u_{2}^{\alpha}-v_{2}^{\alpha}\right|\right\} .
$$

It is well known that $\left(\mathbb{R}_{\mathscr{F}}, d\right)$ is a complete metric space [10]. We list the following properties of $d(u, v)$ :

$$
\begin{aligned}
d(u+w, v+w) & =d(u, v), \\
d(u, v) & =d(v, u), \\
d(k u, k v) & =|k| d(u, v), \\
d(u, v) & \leq d(u, w)+d(w, v),
\end{aligned}
$$

for all $u, v, w \in \mathbb{R}_{\mathscr{F}}$ and $\lambda \in \mathbb{R}$.

Let $T=(0, a] \subset \mathbb{R}$ be a compact interval. We denote by $C\left(T, \mathbb{R}_{\mathscr{F}}\right)$ the space of all continuous fuzzy functions on $T$ and is a complete metric space with respect to the metric

$$
h(u, v)=\sup _{t \in T} d(u(t), v(t)) .
$$

Also, we denote by $L^{1}\left(T, \mathbb{R}_{\mathscr{F}}\right)$ the space of all fuzzy functions $f: T \longrightarrow \mathbb{R}_{\mathscr{F}}$ which are Lebesgue integrable on the bounded interval $T$ of $\mathbb{R}$.

Let $u: T \longrightarrow \mathbb{R}_{\mathscr{F}}$ be a fuzzy function. We denote

$$
[u(t)]^{\alpha}=\left[u_{1}^{\alpha}(t), u_{2}^{\alpha}(t)\right], \quad t \in T, \alpha \in[0,1] .
$$

The derivative $u^{\prime}(t)$ of a fuzzy function $u$ is defined by [11]

$$
\left[u^{\prime}(t)\right]^{\alpha}=\left[\left(u_{1}^{\alpha}\right)^{\prime}(t),\left(u_{2}^{\alpha}\right)^{\prime}(t)\right], \quad \alpha \in[0,1],
$$

provided this equation defines a fuzzy number $u^{\prime}(t) \in \mathbb{R}_{\mathscr{F}}$. The fuzzy integral $\int_{a}^{b} u(t) d t, a, b \in T$ is defined by [12]

$$
\left[\int_{a}^{b} u(t) d t\right]^{\alpha}=\left[\int_{a}^{b} u_{1}^{\alpha}(t) d t, \int_{a}^{b} u_{2}^{\alpha}(t) d t\right]
$$

provided that the Lebesgue integrals on the right exist.

From [13], we have the following theorems:

Theorem 2. There exists a real Banach space $X$ such that $\mathbb{R}_{\mathscr{F}}$ can be the embedding as a convex cone $C$ with vertex 0 into $X$. Furthermore, the following conditions hold:

(i) The embedding $j$ is isometric

(ii) Addition in $X$ induces addition in $\mathbb{R}_{\mathscr{F}}$, i.e., for any $u, v \in \mathbb{R}_{\mathscr{F}}$

(iii) Multiplication by a non-negative real number in $X$ induces the corresponding operation in $\mathbb{R}_{\mathscr{F}}$, i.e., for any $u \in \mathbb{R}_{\mathscr{F}}$

(iv) $C-C$ is dense in $X$

(v) $C$ is closed

Remark 1. Let $\tilde{j}: \mathbb{R}_{\mathscr{F}} \longrightarrow X$ as $\widetilde{j}(u)=j((-1) u), u \in \mathbb{R}_{\mathscr{F}}$. It verifies the following properties:

$$
\begin{aligned}
\|\widetilde{j}(u)-\widetilde{j}(v)\| & =d(u, v), \\
\widetilde{j}(s u+t v) & =\tilde{s} \tilde{j}(u)+t \tilde{j}(v),
\end{aligned}
$$

for all $u, v \in \mathbb{R}_{\mathscr{F}}, t, s \geq 0$,

$$
\begin{aligned}
\tilde{j}\left(\mathbb{R}_{\mathscr{F}}\right)-j\left(\mathbb{R}_{\mathscr{F}}\right) & =C, \\
\text { since }(-1) \mathbb{R}_{\mathscr{F}} & =\mathbb{R}_{\mathscr{F}} .
\end{aligned}
$$

Theorem 3. Let $X$ be a Banach space and $j$ an embedding as in Theorem 2, $u: T \longrightarrow \mathbb{R}_{\mathscr{F}}$, and assume $j \circ u$ is Bochner integrable over $T$. Then, $\int u \in \mathbb{R}_{\mathscr{F}}$, and

$$
j\left(\int u(t) d t\right)=\int j(u(t)) d t .
$$

Remark 2. By the definition of fuzzy integral [14], the above equality yields

$$
j^{-1}\left(\int j(u(t)) d t\right)=\int u(t) d t .
$$




\section{Fuzzy Fractional Integral and Fuzzy Fractional Derivative}

Let $u(t) \in \mathbb{R}_{\mathscr{F}}$ be such that $[u(t)]^{\alpha}=\left[u_{1}^{\alpha}(t), u_{2}^{\alpha}(t)\right]$ for all $t \in T$ and $q \in \mathbb{R}_{+}$. Suppose that $u_{1}^{\alpha}, u_{2}^{\alpha} \in C(T, \mathbb{R}) \cap L^{1}(T, \mathbb{R})$ for all $\alpha \in[0,1]$, and let

$$
\begin{aligned}
A_{\alpha} & :=\frac{1}{\Gamma(q)}\left[\int_{0}^{t}(t-s)^{q-1} u_{1}^{\alpha}(s) d s, \int_{0}^{t}(t-s)^{q-1} u_{2}^{\alpha}(s) d s\right] \\
& :=\left[\phi_{q}(t) * u_{1}^{\alpha}(t), \phi_{q}(t) * u_{2}^{\alpha}(t)\right] .
\end{aligned}
$$

Lemma 2 (see [1]). The family $\left\{A_{\alpha} ; \alpha \in[0,1]\right\}$, given by (16), defined a fuzzy number $u \in \mathbb{R}_{\mathscr{F}}$ such that $[u]^{\alpha}=A_{\alpha}$.

We define

$$
\begin{aligned}
& \phi_{q}(t)= \begin{cases}\frac{t^{q-1}}{\Gamma(q)}, & t>0, \\
0, & t \leq 0,\end{cases} \\
& \phi_{-q}(t)=\phi_{1+k-q}(t) * \delta^{1+k}(t), \quad k=[q], \\
& \phi_{-n}(t)=\delta^{n}(t), \quad n=0,1,2, \ldots,
\end{aligned}
$$

where $\delta^{n}(t)$ is the $n$th derivative of the delta function and $\Gamma(\cdot)$ is the gamma function (for the properties of $\phi_{q}(t)$, see $[15,16])$. These functions satisfy the semigroup property:

$$
\phi_{q}(t) * \phi_{p}(t)=\phi_{q+p}(t), \quad \text { for } p>0 .
$$

The Sobolev spaces can be defined in the following way [17]:

$$
\begin{aligned}
W^{k, p}(T, \mathbb{R}) & =\left\{u \mid \exists \varphi \in L^{p}(T, \mathbb{R}): u(t)\right. \\
& \left.=\sum_{j=0}^{k} C_{j} \frac{t^{j}}{j !}+\frac{t^{k}}{k !} * \varphi(t), \quad t \in T\right\} .
\end{aligned}
$$

Note that $\varphi(t)=u^{k+1}(t), C_{j}=u^{j}(0)$. Let

$$
W_{0}^{k, p}=\left\{u \in W^{k, p}(T, \mathbb{R}) \mid u^{j}(0)=0, \quad j=0,1,2, \ldots, k\right\} .
$$

So, $u \in W_{0}^{k, p}$, if $u(t)=\left(t^{k-1} / k-1 !\right) * \varphi(t)$ for some $\varphi \in L^{1}(T, \mathbb{R})$.

Remark 3. Let $u: T \longrightarrow \mathbb{R}_{\mathscr{F}}$ be such that $[u(t)]^{\alpha}=$ $\left[u_{1}^{\alpha}(t), u_{2}^{\alpha}(t)\right]$ for all $t \in T$ and $\alpha \in[0,1]$. Suppose that $u_{1}^{\alpha}, u_{2}^{\alpha} \in W^{k, 1}(T, \mathbb{R})$ for all $\alpha \in[0,1]$, and let

$$
B_{\alpha}=:\left[\sum_{j=0}^{k}\left(C_{1}^{\alpha}\right)_{j} \frac{t^{j}}{j !}+\frac{t^{k}}{k !} * \varphi_{1}^{\alpha}(t), \sum_{j=0}^{k}\left(C_{2}^{\alpha}\right)_{j} \frac{t^{j}}{j !}+\frac{t^{k}}{k !} * \varphi_{2}^{\alpha}(t)\right]
$$

Note that $\varphi_{i}^{\alpha}(t)=\left(u_{i}^{\alpha}\right)^{(j)}(t),\left(C_{i}^{\alpha}\right)_{j}=\left(u_{i}^{\alpha}\right)^{(j)}(0)$, for $i=1,2$.

According to lemma (2) and by the notation of $\varphi_{i}^{\alpha}$ and $\left(C_{i}^{\alpha}\right)_{j}, i=1,2$, the family $\left\{B_{\alpha}, \alpha \in[0,1]\right\}$, given by $(21)$, defined a fuzzy number $u \in \mathbb{R}_{\mathscr{F}}$ such that $[u]^{\alpha}=B_{\alpha}$.

3.1. Fuzzy Fractional Integral and Derivative. Let $u \in C\left(T, \mathbb{R}_{\mathscr{F}}\right) \cap L^{1}\left(T, \mathbb{R}_{\mathscr{F}}\right)$. Define the fuzzy fractional primitive of order $q>0$ of $u$ :

$$
I^{q} u(t)=\frac{1}{\Gamma(q)} \int_{a}^{t}(t-s)^{q-1} u(s) d s, \quad t \in T,
$$

by

$$
\begin{aligned}
{\left[I^{q} u(t)\right]^{\alpha}=} & \frac{1}{\Gamma(q)}\left[\int_{0}^{t}(t-s)^{q-1} u_{1}^{\alpha}(s) d s,\right. \\
& \left.\int_{0}^{t}(t-s)^{q-1} u_{2}^{\alpha}(s) d s\right], \quad t \in T \\
= & {\left[u_{1}^{\alpha}(t) * \phi_{q}(t), u_{2}^{\alpha}(t) * \phi_{q}(t)\right], \quad t \in T . }
\end{aligned}
$$

For $q=1$, we obtain $I^{1} u(t)=\int_{a}^{t} u(s) d s, t \in T$, that is, the integral operator. Also, the following properties are obvious:

(i) $I^{q}(c u)(t)=c I^{q}(u)(t)$ for each constant $c \in \mathbb{R}_{\mathscr{F}}$

(ii) $I^{q}(u+v)(t)=I^{q}(u)(t)+I^{q}(v)(t)$

Proposition 1. [1]. If $u \in C\left(T, \mathbb{R}_{\mathscr{F}}\right) \cap L^{1}\left(T, \mathbb{R}_{\mathscr{F}}\right)$, then we have

$$
I^{p} I^{q} u=I^{p+q} u, \quad p, q>0
$$

Definition 1. Let $u \in C^{1+k}\left(T, \mathbb{R}_{\mathscr{F}}\right) \cap L^{1}\left(T, \mathbb{R}_{\mathscr{F}}\right)$ be a given function such that $[u]^{\alpha}=\left[u_{1}^{\alpha}, u_{2}^{\alpha}\right]$ for all $t \in T$, and $\alpha \in[0,1]$, the fuzzy fractional differential operator in the Riemann-Liouville sense, is defined for all $u$ satisfying

$$
\begin{aligned}
\phi_{k+1-q} * u & \in W^{k, 1}(T), \\
D^{q} u(t) & =D^{1+k}\left(\phi_{1+k-q} * u\right)(t)=D^{1+k} I^{k+1-q} u(t) \\
& =\frac{1}{\Gamma(1+k-q)} D^{1+k} \int_{0}^{t} u(s)(t-s)^{k-q} d s,
\end{aligned}
$$


by

$$
\begin{aligned}
{\left[D^{q} u(t)\right]^{\alpha}=} & {\left[D^{1+k}\left(\phi_{1+k-q} * u_{1}^{\alpha}\right)(t), D^{1+k}\left(\phi_{1+k-q} * u_{2}^{\alpha}\right)(t)\right] } \\
= & \frac{1}{\Gamma(1+k-q)}\left[D^{1+k} \int_{0}^{t} u_{1}^{\alpha}(s)(t-s)^{k-q} d s,\right. \\
& \left.D^{1+k} \int_{0}^{1} u_{2}^{\alpha}(s)(t-s)^{k-q} d s\right] .
\end{aligned}
$$

In fact,

$\left[D^{q} u(t)\right]^{\alpha}=\left[D^{q} u_{1}^{\alpha}(t), D^{q} u_{2}^{\alpha}(t)\right], \quad$ for all $t \in T$ and $\alpha \in[0,1]$,

and the fuzzy fractional differential operator in the Caputo sense is defined:

$$
\begin{aligned}
D_{c}^{q} u(t) & =D^{1+k} \phi_{1+k-q}(t) * u(t)=I^{k+1-q} D^{1+k} u(t) \\
& =\frac{1}{\Gamma(1+k-q)} \int_{0}^{t} D^{1+k} u(s)(t-s)^{k-q} d s
\end{aligned}
$$

by

$$
\begin{aligned}
{\left[D_{c}^{q} u(t)\right]^{\alpha}=} & {\left[D^{1+k} u_{1}^{\alpha}(t) * \phi_{1+k-q}(t), D^{1+k} u_{2}^{\alpha}(t) * \phi_{1+k-q}(t)\right] } \\
= & \frac{1}{\Gamma(1+k-q)}\left[\int_{0}^{t} D^{1+k} u_{1}^{\alpha}(s)(t-s)^{k-q} d s\right. \\
& \left.\int_{0}^{t} D^{1+k} u_{2}^{\alpha}(s)(t-s)^{k-q} d s\right] .
\end{aligned}
$$

For $k=0$, we obtain $\left[D_{c}^{q} u(t)\right]^{\alpha}=(1 / \Gamma(1-q))$ $\left[\int_{0}^{t}(d / d s) u_{1}^{\alpha}(t)(t-s)^{-q} d s, \int_{0}^{t}(d / d s) u_{2}^{\alpha}(t)(t-s)^{-q} d s\right]$, provided that the equation defines a fuzzy number $D_{c}^{q} u(t) \in \mathbb{R}_{\mathscr{F}}$. In fact,

$\left[D_{c}^{q} u(t)\right]^{\alpha}=\left[D_{c}^{q} u_{1}^{\alpha}(t), D_{c}^{q} u_{2}^{\alpha}(t)\right], \quad$ for all $t \in T$ and $\alpha \in[0,1]$.

Some simple but relevant results valid for $q, p, t>0$ are

$$
I^{q} \phi_{p}=\phi_{q+p}, D^{q} \phi_{p}=\phi_{p-q}, \quad p \geq q .
$$

Proposition 2. Let $q>0$ and $k=[q]$. Then, for any $u \in L^{1}\left(T, \mathbb{R}_{\mathscr{F}}\right)$,

$$
D^{q} I^{q} u(t)=u(t)
$$

If moreover (25) holds, then

$$
I^{q} D^{q} u(t)=u(t)-{ }^{h} \sum_{j=0}^{k}\left(\phi_{k+1-q} * u\right)^{(j)}(0) \phi_{q+j-k}(t) .
$$

Proof. Let $q>0$ and $f: T \longrightarrow \mathbb{R}_{\mathscr{F}}$ be such that $[u(t)]^{\alpha}=$ $\left[u_{1}^{\alpha}(t), u_{2}^{\alpha}(t)\right]$ for all $t \in T$ and $\alpha \in[0,1]$. If $u_{1}^{\alpha}, u_{2}^{\alpha} \in C(T, \mathbb{R}) \cap L^{1}(T, \mathbb{R})$ for all $\alpha \in[0,1]$, then $\left[I^{q} u\right]^{\alpha}$ satisfies (25): $\left[I^{q} u\right]^{\alpha}=\left[\phi_{q} * u\right]^{\alpha} \in L^{1}\left(T, \mathbb{R}_{\mathscr{F}}\right)$ (i.e., $\phi_{q} * u_{1}^{\alpha}$, $\left.\phi_{q} * u_{2}^{\alpha} \in L^{1}(T, \mathbb{R})\right)$ and

$$
\left[\phi_{k+1-q} * I^{q} u\right]^{\alpha}=\left[\phi_{k+1-q} * \phi_{q} * u\right]^{\alpha}=\left[\phi_{k+1} * u\right]^{\alpha} \in W_{0}^{k, 1}(T) .
$$

That is, $\phi_{k+1} * u_{1}^{\alpha}, \phi_{k+1} * u_{2}^{\alpha} \in W_{0}^{k, 1}$. So, we can apply $\left[D^{q} u\right]^{\alpha}$ to $\left[I^{q} u\right]^{\alpha}$ and thanks to the semigroup property (24)

$$
\left[D^{q} I^{q} u\right]^{\alpha}=\left[D^{k+1} I^{k+1-q} I^{q} u\right]^{\alpha}=\left[D^{k+1} I^{k+1} u\right]^{\alpha}=[u]^{\alpha} .
$$

If $u$ satisfies (25), then according to (19),

$$
\left[\phi_{k+1-q} * u\right]^{\alpha}=\left[\sum_{j=0}^{k} C_{j} \phi_{j+1}(t)+\phi_{k+1} * \varphi\right]^{\alpha},
$$

where $\varphi_{1}, \varphi_{2} \in L^{1}(T)$ and $\left[C_{j}\right]^{\alpha}=\left[\left(\phi_{k+1-q} * u\right)^{(j)}(0)\right]^{\alpha}$. Therefore,

$$
\left[I^{q} D^{q} u\right]^{\alpha}=\left[I^{q} D^{k+1} I^{k+1-q} u\right]^{\alpha}=\left[I^{q} D^{k+1} \phi_{k+1-q} * u\right]^{\alpha}=\left[I^{q} \varphi\right]^{\alpha} .
$$

Convolving both sides of (37) with $\phi_{q}$ and applying the semigroup property (18), we obtain

$$
\left[\phi_{k+1} * f\right]^{\alpha}=\left[\sum_{j=0}^{k} C_{j} \phi_{q+j+1}(t)+\phi_{q+k+1} * \varphi\right]^{\alpha} .
$$

An application of $D^{k+1}$ to both sides gives

$$
\begin{aligned}
{[u]^{\alpha}=} & {\left[\sum_{j=0}^{k} C_{j} \phi_{q+j-k}(t)+\phi_{q} * \varphi\right]^{\alpha}=\left[\sum_{j=0}^{k} C_{j} \phi_{q+j-k}(t)\right]^{\alpha} } \\
& +\left[\phi_{q} * \varphi\right]^{\alpha},
\end{aligned}
$$

and then,

$$
[u]^{\alpha}-^{h}\left[\sum_{j=0}^{k} C_{j} \phi_{q+j+1-k}(t)\right]^{\alpha}=\left[\phi_{q} * \varphi\right]^{\alpha},
$$

which together with (38) implies (34). If $\phi_{k+1-q} * u_{1}^{\alpha}$, $\phi_{k+1-q} * u_{2}^{\alpha} \in W_{0}^{k, 1}(T)$, that is, $C_{j}=0, j=0,1, \ldots, k$, we have $I^{q} D^{q} u=u$.

Remark 4

$$
D^{q} u(t)=\sum_{j=0}^{k} u^{j}(0) \phi_{j-q}(t)+I^{k+1-q} D^{k+1} u(t),
$$

and then, 


$$
\begin{aligned}
D_{c}^{q} u(t) & =D^{q} u(t)-^{h} \sum_{j=0}^{k} u^{j}(0) \phi_{j-q}(t) \\
& =D^{q}\left(u(t){ }^{h} \sum_{j=0}^{k} u^{j}(0) \phi_{j}(t)\right) .
\end{aligned}
$$

Therefore,

$$
\begin{aligned}
& D_{c}^{q} I^{q} u(t)=u(t), \\
& I^{q} D_{c}^{q} u(t)=u(t)-{ }^{h} \sum_{j=0}^{k} u^{j}(0) \phi_{j},
\end{aligned}
$$

and the first identity is valid for all $u_{1}^{\alpha}, u_{2}^{\alpha} \in L^{1}(T)$, the second for $u_{1}^{\alpha}, u_{2}^{\alpha} \in C^{k}(T)$.

3.2. Fuzzy Laplace Transform. Let $u(t) \in \mathbb{R}_{\mathscr{F}}$ be such that $[u(t)]^{\alpha}=\left[u_{1}^{\alpha}(t), u_{2}^{\alpha}(t)\right]$ for all $t \in T$. Suppose that $u_{1}^{\alpha}, u_{2}^{\alpha} \in C(T, \mathbb{R}) \cap L^{1}(T, \mathbb{R})$ for all $\alpha \in[0,1]$. We define the fuzzy Laplace transform [18] by

$$
\begin{aligned}
{[\mathscr{L} u(\lambda)]^{\alpha} } & =\left[\int_{0}^{\infty} \exp (-\lambda t) u_{1}^{\alpha}(t) d t, \int_{0}^{\infty} \exp (-\lambda t) u_{2}^{\alpha}(t) d t\right] \\
& =\left[L u_{1}^{\alpha}(\lambda), L u_{2}^{\alpha}(\lambda)\right],
\end{aligned}
$$

where $\lambda>0$ and real.

Theorem 4. [18]. Let $u$ and $v$ are continuous fuzzy-valued functions. Suppose that $c_{1}$ and $c_{2}$ are constants. Then,

$$
\left[\mathscr{L}\left(\left(c_{1} u(\lambda)\right)+\left(c_{2} v(\lambda)\right)\right)\right]^{\alpha}=c_{1}[\mathscr{L} u(\lambda)]^{\alpha}+c_{2}[\mathscr{L} v(\lambda)]^{\alpha},
$$

Lemma 3. Let $u$ is continuous fuzzy-valued function and $c \in \mathbb{R}:$

$$
[\mathscr{L} c u(\lambda)]^{\alpha}=c[\mathscr{L} u(\lambda)]^{\alpha}
$$

As in [5], we can introduce laplace transforms of derivatives by:

Proposition 3. Let $u \in C\left(T, \mathbb{R}_{\mathscr{F}}\right) \cap L^{1}\left(T, \mathbb{R}_{\mathscr{F}}\right)$, and then

$$
\begin{aligned}
& \mathscr{L} D^{q} u(\lambda)=\lambda^{q} \mathscr{L} u(\lambda){ }^{h} \sum_{j=0}^{k}\left(\phi_{k+1-q} * u\right)^{(j)}(0) \lambda^{k-j}, \\
& \mathscr{L} D_{c}^{q} u(\lambda)=\lambda^{q} \mathscr{L} u(\lambda)-{ }^{h} \sum_{j=0}^{k}(u)^{(j)}(0) \lambda^{q-j} .
\end{aligned}
$$

Proof. We prove (49) because the proof of (48) is similar. For arbitrary fixed $\alpha \in[0,1]$, we have

$$
\begin{gathered}
\lambda^{q}[\mathscr{L} u(\lambda)]^{\alpha}-^{h} \sum_{j=0}^{k}\left[(u)^{(j)}(0)\right]^{\alpha} \lambda^{q-j} \\
=\left[\lambda^{q} L u_{1}^{\alpha}(\lambda)-\sum_{j=0}^{k}\left(u_{1}^{\alpha}\right)^{(j)}(0) \lambda^{q-j},\right. \\
\left.\lambda^{q} L u_{2}^{\alpha}(\lambda)-\sum_{j=0}^{k}\left(u_{2}^{\alpha}\right)^{(j)}(0) \lambda^{q-j}\right] .
\end{gathered}
$$

We apply the properties of the Laplace transform, and since $L \phi_{q}(\lambda)=\lambda^{-q}$, we obtain

$$
\begin{aligned}
& L D_{c}^{q} u_{1}^{\alpha}(\lambda)=\lambda^{q} L u_{1}^{\alpha}(\lambda)-\sum_{j=0}^{k}\left(u_{1}^{\alpha}\right)^{(j)}(0) \lambda^{q-j}, \\
& L D_{c}^{q} u_{2}^{\alpha}(\lambda)=\lambda^{q} L u_{2}^{\alpha}(\lambda)-\sum_{j=0}^{k}\left(u_{2}^{\alpha}\right)^{(j)}(0) \lambda^{q-j} .
\end{aligned}
$$

Then, we conclude that

$$
\lambda^{q}[\mathscr{L} u(\lambda)]^{\alpha}-^{h} \sum_{j=0}^{k}\left[(u)^{(j)}(0)\right]^{\alpha} \lambda^{q-j}=\left[L D_{c}^{q} u_{1}^{\alpha}(\lambda), L D_{c}^{q} u_{2}^{\alpha}(\lambda)\right] \text {, }
$$

by linearity of $L$,

$$
\lambda^{q}[\mathscr{L} u(\lambda)]^{\alpha}-^{h} \sum_{j=0}^{k}\left[(u)^{(j)}(0)\right]^{\alpha} \lambda^{q-j}=L\left[D_{c}^{q} u_{1}^{\alpha}(\lambda), D_{c}^{q} u_{2}^{\alpha}(\lambda)\right] .
$$

Using (31) leads to obtain

$$
\lambda^{q}[\mathscr{L} f(\lambda)]^{\alpha}-^{h} \sum_{j=0}^{k}\left[(f)^{(j)}(0)\right]^{\alpha} \lambda^{q-j}=\left[\mathscr{L}\left(D_{c}^{q} f(\lambda)\right)\right]^{\alpha} .
$$

3.3. Fuzzy Solution Operators. We adopt the general definition and theorem of operator theory on $\mathbb{R}_{\mathscr{F}}$ in [6]. Let $A: \mathbb{R}_{\mathscr{F}} \longrightarrow \mathbb{R}_{\mathscr{F}}$ is linear if

$$
\left\{\begin{array}{l}
A(x+y)=A(x)+A(y), \\
A(k \cdot x)=k \cdot A(x)
\end{array}\right.
$$

for all $x, y \in L\left(\mathbb{R}_{\mathscr{F}}\right), k \in \mathbb{R}$, where $L\left(\mathbb{R}_{\mathscr{F}}\right)$ is $A \in L^{+}\left(\mathbb{R}_{\mathscr{F}}\right)$ and is continuous at each $x \in \mathbb{R}_{\mathscr{F}}$ and $L^{+}\left(\mathbb{R}_{\mathscr{F}}\right)$ is semilinear and continuous at $\widetilde{0}$.

Let us consider the metric $\Phi: L^{+}\left(\mathbb{R}_{\mathscr{F}}\right) \times L^{+}\left(\mathbb{R}_{\mathscr{F}}\right)$ $\longrightarrow \mathbb{R}_{+}$

$$
\Phi(A, B)=\sup \left\{d(A x, B x) ;\|x\|_{\mathscr{F}} \leq 1\right\}
$$

where $\|x\|_{\mathscr{F}}=d(\widetilde{0}, x)$ and we have $\Phi(A, \widetilde{O})=|A|_{\mathscr{F}}$, where $\widetilde{O}: \mathbb{R}_{\mathscr{F}} \longrightarrow \mathbb{R}_{\mathscr{F}}$ is given by $\widetilde{O}(x)=\widetilde{0}, \forall x \in \mathbb{R}_{\mathscr{F}}$.

Theorem 5. Let $A$ be a bounded linear operator on $\mathbb{R}_{\mathscr{F}}[6]$. If $|A|<1$, then $i-A$ is invertible; moreover, 


$$
\lim _{n=\infty} \Phi\left((i-A)^{-1}, \sum_{k=0}^{n} A^{k}\right)=0
$$

Here, $i: \mathbb{R}_{\mathscr{F}} \longrightarrow \mathbb{R}_{\mathscr{F}}$ denotes the identity function of $\mathbb{R}_{\mathscr{F}}$.

Proof. Recall that $\mid\|A\| \|_{\mathscr{F}}=\sup \left\{d(A x, \widetilde{0}), x \in \mathbb{R}_{\mathscr{F}},\|x\|_{\mathscr{F}} \leq 1\right\}$ $\forall x \in \mathbb{R}_{\mathscr{F}}$ Setting $P_{n} x=\sum_{n=0}^{\infty} A^{n} x$ by (Theorem 3.5 and Corollary 3.6 in [6]), it suffices to show that $P_{n} x$ is a Cauchy sequence in the complete metric space $\left(L\left(\mathbb{R}_{\mathscr{F}}\right), \Phi\right)$. First $A \in L\left(\mathbb{R}_{\mathscr{F}}\right)$, it follows $A^{n} \in L\left(\mathbb{R}_{\mathscr{F}}\right)$ for $n=0,1,2, \ldots$ Then for $m<n$ we have

$$
\begin{aligned}
d\left(P_{n} x, P_{m} x\right) & =d\left(\sum_{j=0}^{n} A^{j} x, \sum_{j=0}^{m} A^{j} x\right) \\
& \leq \sum_{j=m}^{n} d\left(\widetilde{0}, A^{j} x\right) .
\end{aligned}
$$

However,

$$
d(\widetilde{0}, A(A x))=\left\|A^{2} x\right\|_{\mathscr{F}} \leq\left.\|\| A\left\|\left.\right|_{\mathscr{F}}\right\| A x\left\|_{\mathscr{F}} \leq\right\|\|A\|\right|_{\mathscr{F}} ^{2}\|x\|_{\mathscr{F}},
$$

and, so by induction, one can obtain

$$
\left\|A^{j}\right\|_{\mathscr{F}} \leq|\|A\||_{\mathscr{F}}^{j}, \quad \text { for all } j=2,3, \ldots
$$

We obtain

$$
d\left(P_{n} x, P_{m} x\right) \leq \sum_{j=m}^{n}\left(|\|A\||_{\mathscr{F}}\|x\|_{\mathscr{F}}\right)^{j},
$$

and passing to supremum with $\|x\|_{\mathscr{F}} \leq 1$ we get

$$
\begin{aligned}
\Phi\left(P_{n}, P_{m}\right) & \leq \sum_{j=m}^{n}|\|A\||_{\mathscr{F}}^{j} \\
& \leq \sum_{j=m}^{+\infty}|\|A\||_{\mathscr{F}}^{j}=\frac{\|\| A \|\left.\right|_{\mathscr{F}} ^{m}}{1-\|A\|_{\mathscr{F}}} \longrightarrow 0 \text { as } m \longrightarrow \infty .
\end{aligned}
$$

Therefore, $\left(P_{n}\right)_{n \geq 0}$ is a uniform Cauchy sequence on $[-a, a], a>0$.

$$
\begin{aligned}
(i-A)\left(\sum_{n=0}^{\infty} A^{n}\right) & =\sum_{n=0}^{\infty} A^{n}-\sum_{n=0}^{\infty} A^{n+1} \\
& =i+\sum_{n=1}^{\infty} A^{n}-\sum_{n=1}^{\infty} A^{n}=i,
\end{aligned}
$$

and $\left(\sum_{n=0}^{\infty} A^{n}\right)(i-A)=i$. It now follows that $(i-A)^{-1}=\sum_{n=0}^{\infty} A^{n}$; therefore, its limit $(i-A)^{-1}$ exists in $L\left(\mathbb{R}_{\mathscr{F}}\right)$. So, $\lim _{n=\infty} \Phi\left((i-A)^{-1}, P_{n}\right)=0$ holds uniformly on each compact interval $[-a, a], a>0$.

Motivated by the above definitions in $[19,20]$, we can give the following definition.
Definition 2. The resolvent set of $\mathrm{A}$, denoted by $\rho(A)$, is the set of all real numbers $\lambda^{q}$ such that $\lambda^{q} i-A$ is bijective, i.e.,

$$
\rho(A)=\left\{\lambda^{q} \in \mathbb{R}_{*}^{+}:\left(\lambda^{q} i-A\right)^{-1} \text { exists }\right\} .
$$

Remark 5. Let $\left|\lambda^{q}\right|>|\|A\||$. Then, $\left\|\left|\left(A / \lambda^{q}\right)\right|\right\|<1$. It follows from Theorem 5 that $\left(i-\left(A / \lambda^{q}\right)\right)^{-1}$ exists, and

$$
R\left(\lambda^{q}, A\right)=\left(\lambda^{q} i-A\right)^{-1}=\frac{1}{\lambda^{q}}\left(i-\frac{A}{\lambda^{q}}\right)^{-1} .
$$

Lemma 4. Let $A: \mathbb{R}_{\mathscr{F}} \longrightarrow \mathbb{R}_{\mathscr{F}}$ and $A_{1}=j A j^{-1}: C \longrightarrow C$ tow operator.

$A$ is the operator of the resolvent $R\left(\lambda^{q}, A\right)$ on $\mathbb{R}_{\mathscr{F}}$ if and only if $A_{1}$ is the operator of the resolvent $R_{1}\left(\lambda^{q}, A_{1}\right)$ defining on the convex closed set $C$ and $R_{1}\left(\lambda^{q}, A_{1}\right)=j R\left(\lambda^{q}, A\right) j^{-1}$ and $\Phi\left(R\left(\lambda^{q}, A\right), \widetilde{O}\right) \leq\left(M /\left|\lambda^{q}-\omega\right|\right)$.

Proof. We assume that $\Phi\left(R\left(\lambda^{q}, A\right), \widetilde{O}\right) \leq\left(M /\left|\lambda^{q}-\omega\right|\right)$ on $\mathbb{R}_{\mathscr{F}}$. By the properties of $j$ we have, for all $u \in C$,

$$
\begin{aligned}
\left\|R_{1}\left(\lambda^{q}, A_{1}\right) u\right\| & =\left\|j R\left(\lambda^{q}, A\right) j^{-1} u\right\|=d\left(R\left(\lambda^{q}, A\right) j^{-1} u, j^{-1} 0\right), \\
d\left(j^{-1} u, j^{-1} 0\right) & =\|u\| .
\end{aligned}
$$

It follows that

$$
\begin{aligned}
\sup _{u \leq 1}\left\|R_{1}\left(\lambda^{q}, A_{1}\right) u\right\| & =\sup _{\|x\|_{\mathscr{F}} \leq 1} d\left(R\left(\lambda^{q}, A\right) x, \widetilde{0}\right) \\
\left\|R_{1}\left(\lambda^{q}, A_{1}\right)\right\| & =\Phi\left(d\left(R\left(\lambda^{q}, A\right)\right), \widetilde{O}\right) \leq \frac{M}{\left|\lambda^{q}-\omega\right|} .
\end{aligned}
$$

Conversely, if $\left\|R_{1}\left(\lambda^{q}, A_{1}\right)\right\| \leq\left(M /\left|\lambda^{q}-\omega\right|\right)$ on $C$, then for all $x \in \mathbb{R}_{\mathscr{F}}$

$$
\begin{aligned}
d\left(R\left(\lambda^{q}, A\right) x, \widetilde{0}\right) & =d\left(j^{-1} R_{1}\left(\lambda^{q}, A_{1}\right) j x, j^{-1} \tilde{0}\right)=\left\|R_{1}\left(\lambda^{q}, A_{1}\right) j x\right\|, \\
\|j x\| & =d(x, \widetilde{0}) .
\end{aligned}
$$

It follows that

$$
\begin{aligned}
\sup _{\|x\|_{\mathscr{F}} \leq 1} d\left(R\left(\lambda^{q}, A\right) x, \widetilde{0}\right) & =\sup _{\|u\| \leq 1}\left\|R_{1}\left(\lambda^{q}, A_{1}\right) u\right\|, \\
\Phi\left(R\left(\lambda^{q}, A\right), \widetilde{O}\right) & =\left\|R_{1}\left(\lambda^{q}, A_{1}\right)\right\| \leq \frac{M}{\left|\lambda^{q}-\omega\right|},
\end{aligned}
$$

which completes the proof.

Next, we need to define the fuzzy solution operator (or fuzzy $q$-resolvent family), which is similar to that given in [19].

Consider the following particular case of (82) for the Caputo fractional derivative evolution equation of order $q(k<q<k+1)$ is an integer:

$$
\begin{cases}D^{q} u(t)=A u(t), & t \in T, q \in \mathbb{R}_{+}, \\ u(0)=u_{j},\left.D^{j} u(t)\right|_{t=0}=u_{j}(0)=0, & j=1,2,3, \ldots, k\end{cases}
$$


where $A: \mathbb{R}_{\mathscr{F}} \longrightarrow \mathbb{R}_{\mathscr{F}}$.

Applying (44), we obtain that the Cauchy problem (26)

$$
u(t)=u_{0}+\frac{1}{\Gamma(q)} \int_{0}^{t}(t-s)^{q} A u(s) d s,
$$

and then we define the solution operator of (70) in terms of the corresponding integral equation (71).

Definition 3. A family of bounded linear operators $\left\{S_{q}(t)\right\}_{t \geq 0}$ on $\mathbb{R}_{\mathscr{F}}$ is called a solution operator for (71) (or $q$ resolvent family), if the following conditions are satisfied:

(1) $S_{q}(t)$ is strongly continuous for $t \geq 0$ and $S_{q}(0)=i$, the identity mapping on $\mathbb{R}_{\mathscr{F}}$

(2) $S_{q}(t) D(A) \subset D(A)$ and $A S_{q}(t) u_{0}=S_{q}(t) A u_{0}$ for all $u_{0} \in D(A), t \geq 0$

(3) $S_{q}(t) u_{0}$ is a solution of (71) for all $u_{0} \in D(A), t \geq 0$

Definition 4. The solution operator $S_{q}(t)$ is called exponentially bounded if there are constants $M \geq 1$ and $w \geq 0$ such that

$$
\Phi\left(S_{q}(t), \widetilde{O}\right) \leq M e^{w t}, \quad t \geq 0 .
$$

Proposition 4. If $S_{q}(t)$ is the solution operator of (70) and $u_{0} \in \mathbb{R}_{\mathscr{F}}$, then if for $t>0$, the Hukuhara difference $S_{q}(t) u_{0}-{ }^{h} u_{0}$ exists, we define

$$
A u_{0}=\Gamma(1+q) \lim _{t \rightarrow 0} \frac{S_{q}(t) u_{0}-{ }^{h} u_{0}}{t^{q}},
$$

which this limit exists in the metric space $\left(\mathbb{R}_{\mathscr{F}}, d\right)$.

Proof. Let $v(\cdot) \in C\left(T, \mathbb{R}_{\mathscr{F}}\right)$, we have

$$
\begin{aligned}
v(t) & =\phi_{-1} * v(t)=\left(\phi_{q} \phi_{-1-q}\right) * v(t)=\phi_{q} * v(t) * \phi_{-1-q} \\
& =\Gamma(1+q) \frac{I^{q} v(t)}{t^{q}} .
\end{aligned}
$$

Taking $v(t)=\left(D^{q} S_{q}\right)(t) u_{0}$ and using (70) and (44), we obtain (73)

Lemma 5. Let $A: \mathbb{R}_{\mathscr{F}} \longrightarrow \mathbb{R}_{\mathscr{F}}$ and $A_{1}=j A j^{-1}: C \longrightarrow C$ tow operator.

$A$ is the generator of a fuzzy q-resolvent family $\left\{S_{q}(t)\right\}_{t \geq 0}$ on $\mathbb{R}_{\mathscr{F}}$ if and only if $A_{1}$ is the generator of an q-resolvent family $\left\{S_{q}^{1}(t)\right\}_{t \geq 0}$ defining on the convex closed set $C$ by $S_{q}^{1}=j S_{q}(t) j^{-1}$.

Proof. Follows from the definition of $\left\{S_{q}(t)\right\}_{t>0}$ and $\left\{S_{q}^{1}(t)\right\}_{t \geq 0}$ (see [19]), then $\left\{S_{q}(t)\right\}_{t \geq 0}$ is the fuzzy solution operator on $\mathbb{R}_{\mathscr{F}}$ if and only if $\left\{S_{q}^{1}(t)\right\}_{t \geq 0}$ is the solution operator on $C$.
We assume that $A$ is the generator of a fuzzy $q$-resolvent family $\left\{S_{q}(t)\right\}_{t>0}$ on $\mathbb{R}_{\mathscr{F}}$. By the properties of $j$ we have, for all $u_{0} \in j^{-1}(D(A))$,

$$
\begin{aligned}
\Gamma(q+1) \lim _{t \rightarrow 0} \frac{S_{q}^{1}(t) u_{0}-u_{0}}{t^{q}} & =\Gamma(q+1) \lim _{t \rightarrow 0} \frac{j S_{q}(t) j^{-1} u_{0}-j j^{-1} u_{0}}{t^{q}} \\
& =j\left(\Gamma(q+1) \lim _{t \rightarrow 0} \frac{S_{q}(t) j^{-1} u_{0}{ }^{h} j^{-1} u_{0}}{t^{q}}\right) \\
& =j A j^{-1} u_{0}=A_{1} u_{0} .
\end{aligned}
$$

Conversely, if $A_{1}$ is the generator of a $q$-resolvent family $\left\{S_{q}^{1}(t)\right\}_{t \geq 0}$ on $C$, then for all $u_{0} \in D(A)$

$$
\begin{aligned}
\Gamma(q+1) \lim _{t \rightarrow 0} \frac{S_{q}(t) u_{0}-^{h} u_{0}}{t^{q}} & =\Gamma(q+1) \lim _{t \rightarrow 0} \frac{j^{-1} S_{q}^{1}(t) j u_{0}-^{h} j^{-1} j u_{0}}{t^{q}} \\
& =j^{-1}\left(\Gamma(q+1) \lim _{t \longrightarrow 0} \frac{S_{q}^{1} j u_{0}-{ }^{h} j u_{0}}{t^{q}}\right) \\
& =j^{-1} A_{1} j u_{0}=A u_{0} .
\end{aligned}
$$

Lemma 6. Let $A$ is a operator in (70) and $j$ an embedding as in Theorem 2, the solution operators $S_{q}(t)$ of $(70)$ is defined by

$$
S_{q}(t)=\mathscr{L}^{-1}\left(\lambda^{q-1} R\left(\lambda^{q}, A\right)\right)(t) .
$$

Proof. Taking the fuzzy Laplace transform of (70) on both sides, we obtain

$$
\lambda^{q} \mathscr{L}(u(t))(\lambda)-{ }^{h} \lambda^{q-1} u_{0}=A \mathscr{L}(u(t))(\lambda),
$$

and using the $j$,

$$
\begin{aligned}
\lambda^{q} j(\mathscr{L}(u(t))(\lambda))-j(A \mathscr{L}(u(t))(\lambda)) & =\lambda^{q-1} j\left(u_{0}\right), \quad \text { where } \lambda^{q}>0, \\
\lambda^{q} j(\mathscr{L}(u(t))(\lambda))-j\left(j^{-1} A_{1} j \mathscr{L}(u(t))(\lambda)\right) & =\lambda^{q-1} j\left(u_{0}\right), \\
\left(\lambda^{q} I-A_{1}\right) j(\mathscr{L}(u(t))(\lambda)) & =\lambda^{q-1} j\left(u_{0}\right), \\
\left(\lambda^{q} I-A_{1}\right) L(j(u(t))(\lambda)) & =\lambda^{q-1} j\left(u_{0}\right) .
\end{aligned}
$$

Since $\left(\lambda^{q} I-A_{1}\right)^{-1}$ exist, i.e, $\lambda^{q} \in \rho\left(A_{1}\right)$ (see [20]), from the above equation, we obtain

$$
L(j(u(t))(\lambda))=\left(\lambda^{q} I-A_{1}\right)^{-1} \lambda^{q-1} j\left(u_{0}\right) .
$$

Now (87) follows easily by taking the inverse of Laplace transform and applying $j^{-1}$

$$
\begin{aligned}
j(u(t)) & =L^{-1}\left(\lambda^{q-1} R_{1}\left(\lambda^{q}, A_{1}\right)\right) j\left(u_{0}\right), \\
u(t) & =\mathscr{L}^{-1}\left(\lambda^{q-1} j^{-1} R_{1}\left(\lambda^{q}, A_{1}\right) j\left(u_{0}\right)\right) .
\end{aligned}
$$

This completes the proof. 


\section{Fuzzy Fractional Differential Equations}

Consider the following fuzzy fractional differential equation:

$$
\begin{cases}D^{q} u(t)=A u(t)+f(t, u(t)), & t \in T, q \in \mathbb{R}_{+}, \\ \left.D^{j} u(t)\right|_{t=0}=u_{j}(0), & j=0,1,2, \ldots, k=[q],\end{cases}
$$

where $A$ generator of $q$-resolvent family $\left(S_{q}(t)\right)_{t \geq 0}$ on $\mathbb{R}_{\mathscr{F}}$, $D^{q}$ is the fuzzy caputo fractional differential operator define by (29) and $f \in T \times \mathbb{R}_{\mathscr{F}} \longrightarrow \mathbb{R}_{\mathscr{F}}$ is continuous.

Firstly, we consider the following Cauchy problem

$$
\begin{cases}D^{q} u(t)=A u(t), & t \in T, q \in \mathbb{R}_{+}, \\ \left.D^{j} u(t)\right|_{t=0}=u_{j}(0), & j=0,1,2, \ldots, k=[q] .\end{cases}
$$

The roblem (70) is particular case of (83), and if (70) has a solution operator $S_{q}(t)$, then the corresponding problem (83) is uniquely solvable with the solution

$$
u(t)=\sum_{j=0}^{k}\left(I^{j} S_{q}\right)(t) u_{j}
$$

provided $u_{j} \in D(A), j=0,1,2, \ldots, k$. For this reason, we restrict ourselves to problem (71) (in crisp, see [19]).

Next, we consider the particular case of (82).

$$
\begin{cases}D^{q} u(t)=A u(t)+f(t, u(t)), & t \in T, q \in \mathbb{R}_{+}, \\ \left.D^{j} u(t)\right|_{t=0}=u_{0},\left.D^{j} u(t)\right|_{t=0}=0, & j=1,2, \ldots, k=[q],\end{cases}
$$

where $A$ is a operator and $f$ is an abstract function defined on $T \times \mathbb{R}_{\mathscr{F}}$ and with values in $\mathbb{R}_{\mathscr{F}}$.

Theorem 6. Let $A$ is an operator, $f:=T \times \mathbb{R}_{\mathscr{F}} \longrightarrow \mathbb{R}_{\mathscr{F}}$ be continuous on $T$ and if $f$ satisfies a Hölder condition with an exponent of $\beta \in(0,1]$

$$
d(f(u), f(v)) \leq C(d(u, v))^{\beta} .
$$

The function $u(t) \in C\left(T, \mathbb{R}_{\mathscr{F}}\right)$ is a solution of (85) if and only if

$$
u(t)=S_{q}(t) u_{0}+\int_{0}^{t} T_{q}(t-s) f(s, u(s)) d s
$$

where

$$
\begin{aligned}
& S_{q}(t)=\mathscr{L}^{-1}\left(\lambda^{q-1} R\left(\lambda^{q}, A\right)\right)(t), \\
& T_{q}(t)=\mathscr{L}^{-1}\left(R\left(\lambda^{q}, A\right)\right)(t) .
\end{aligned}
$$

Proof. Now applying the fuzzy Riemann-Liouville fractional integral operator (23) in (85) on both sides, we get

$$
\begin{aligned}
u(t)-{ }^{h} u_{0} & =\phi_{q} * A u(t)+\phi_{q} * f(t, u(t)), \\
u(t) & =u_{0}+\phi_{q} * A u(t)+\phi_{q} * f(t, u(t)),
\end{aligned}
$$

and taking the fuzzy Laplace transform of (89) on both sides, we obtain

$$
\begin{aligned}
\mathscr{L}(u(t))(\lambda)= & \mathscr{L}\left(u_{0}\right)(\lambda)+\mathscr{L}\left(\phi_{q} * A u(t)\right)(\lambda) \\
& +\mathscr{L}\left(\phi_{q} * f(t, u(t))\right)(\lambda),
\end{aligned}
$$

$\lambda^{q} \mathscr{L}(u(t))(\lambda)-{ }^{h} A \mathscr{L}(u(t))(\lambda)=\lambda^{q-1} u_{0}+\mathscr{L}(f(t, u(t)))(\lambda)$.

By using $j$ and if $\left(\lambda^{q} I-A_{1}\right)^{-1}$ exists, i.e., $\lambda^{q} \in \rho\left(A_{1}\right)$, from the above equation, we obtain

$$
\begin{aligned}
j(\mathscr{L}(u(t))(\lambda))= & \left(\lambda^{q} I-A_{1}\right)^{-1} \lambda^{q-1} j\left(u_{0}\right) \\
& +\left(\lambda^{q} I-A_{1}\right)^{-1} j(\mathscr{L}(f(t, u(t)))(\lambda)), \\
L(j(u(t))(\lambda))= & \left(\lambda^{q} I-A_{1}\right)^{-1} \lambda^{q-1} j\left(u_{0}\right) \\
& +\left(\lambda^{q} I-A_{1}\right)^{-1} L(j(f(t, u(t)))(\lambda)) .
\end{aligned}
$$

Now, (87) follows easily by taking the inverse of Laplace transform:

$$
\begin{aligned}
j(u(t))= & L^{-1}\left(\lambda^{q} I-A_{1}\right)^{-1} \lambda^{q-1} j\left(u_{0}\right) \\
& +\int_{0}^{t} L^{-1}\left(\lambda^{q} I-A_{1}\right)^{-1} j(f(s, u(s))) d s,
\end{aligned}
$$

and applying $j^{-1}$ and Lemma 4,

$$
\begin{aligned}
u(t)= & \mathscr{L}^{-1}\left(\lambda^{q} I-A\right)^{-1} \lambda^{q-1} u_{0} \\
& +\int_{0}^{t} \mathscr{L}^{-1}\left(\lambda^{q} I-A_{1}\right)^{-1} f(s, u(s)) d s .
\end{aligned}
$$

This completes the proof.

Theorem 7. Let $A$ be a operator. If satisfies (86), then the solutions of the Cauchy problem (82) are fixed points of the operator equation:

$$
\varphi u(t)=\sum_{j=0}^{k}\left(I^{j} S_{q}\right)(t) u_{j}+\int_{0}^{t} T_{q}(t-s) f(s, u(s)) d s .
$$

Theorem 7 leads to the following appropriate definition of a mild solution to (82).

Definition 5. A function $u(t) \in C\left(T, \mathbb{R}_{\mathscr{F}}\right)$ is called a mild solution of (82), if it satisfies the operator equation:

$$
u(t)=\sum_{j=0}^{k}\left(I^{j} S_{q}\right)(t) u_{j}+\int_{0}^{t} T_{q}(t-s) f(s, u(s)) d s .
$$

Theorem 8. Let $A$ is a operator and $f: T \times \mathbb{R}_{\mathscr{F}} \longrightarrow \mathbb{R}_{\mathscr{F}}$ be continuous on T. Assume that

$\left(H_{0}\right)$ there exists $M \geq 1$ and $\omega>0$ such that

$$
d\left(T_{q} v(t), T_{q} w(t)\right) \leq M e^{\omega t} d(v(t), w(t)), \quad t \in T
$$

$\left(H_{1}\right)$ there exists a constant $L>0$, such that 


$$
d(f(t, v(t)), f(t, w(t))) \leq L d(v(t), w(t)),
$$

for every $v(t), w(t) \in \mathbb{R}_{\mathscr{F}}, t \in T$. Then, problem (82) has a unique mild solution $u(t) \in C\left(T, \mathbb{R}_{\mathscr{F}}\right)$.

Proof. Let $\psi: C\left(T, \mathbb{R}_{\mathscr{F}}\right) \longrightarrow C\left(T, \mathbb{R}_{\mathscr{F}}\right)$ be the operator defined by

$$
\psi u(t)=\sum_{j=0}^{k}\left(I^{j} S_{q}\right)(t) u_{j}+\int_{0}^{t} T_{q}(t-s) f(s, u(s)) d s .
$$

We have to show that $u$ is mild solution of (82) if and only if $\psi u=u$ and $v(t), w(t) \in C\left(T, \mathbb{R}_{\mathscr{F}}\right)$, we find that

$$
\begin{aligned}
d(\psi v(t), \psi w(t))= & d\left(\int_{0}^{t} T_{q}(t-s) f(s, v(s)) d s,\right. \\
& \left.\int_{0}^{t} T_{q}(t-s) f(s, w(s)) d s\right) \\
\leq & \int_{0}^{t} d\left(T_{q}(t-s) f(s, v(s)),\right. \\
& \left.T_{q}(t-s) f(s, w(s))\right) d s \\
\leq & M \int_{0}^{t} e^{\omega(t-s)} d(f(s, v(s)), f(s, w(s))) d s \\
\leq & L e^{\omega a} \int_{0}^{t} d(v(s), w(s)) d s \leq L t e^{\omega a} h(v, w) .
\end{aligned}
$$

We can deduce that

$$
\begin{aligned}
d\left(\psi^{2} v(t), \psi^{2} w(t)\right) & =L e^{\omega a} \int_{0}^{t} d(\psi v(t), \psi w(t)) d s \\
& \leq L e^{\omega a} \int_{0}^{t} s L e^{\omega a} h(v, w) d s \\
& \leq \frac{\left(L e^{\omega a} t\right)^{2}}{2 !} h(v, w) \\
\vdots & \vdots \\
d\left(\psi^{n} v(t), \psi^{n} w(t)\right) & =\frac{\left(L e^{\omega a} t\right)^{n}}{n !} h(v, w) \\
& \leq \frac{\left(L e^{\omega a} a\right)^{n}}{n !} h(v, w),
\end{aligned}
$$

and it follows that

$$
h\left(\psi^{n} v, \psi^{n} w\right)=\frac{\left(L e^{\omega a} t\right)^{n}}{n !} h(v, w), \quad \forall v, w \in C\left(T, \mathbb{R}_{\mathscr{F}}\right) .
$$

Since $\quad\left(\left(L e^{\omega a} a\right)^{n} / n !\right) \longrightarrow 0, \quad \exists p \in \mathbb{N}$ such that $\left(\left(L e^{\omega a} a\right)^{p} / p !\right)<1$.

It follows that $\psi^{p}$ is a contraction, and then $\exists ! u \in C\left(T, \mathbb{R}_{\mathscr{F}}\right)$ such that $\psi^{p} u=u$.

We prove $\psi u=u$. $\psi^{p}(u)=u$ implies that $\psi^{p+1}(u)=\psi u$, one can write $\psi^{P}(\psi u)=\psi u$ it follows that $\psi u$ is a fixed point of $\psi^{p}$, and since the fixed point is unique, we get $\psi(u)=u$.

Hence, (85) has a unique solution.

\section{Data Availability}

The data used to support the findings of this study are available from the corresponding author upon request.

\section{Conflicts of Interest}

The authors declare that they have no conflicts of interest.

\section{References}

[1] S. Arshad and V. Lupulescu, "On the fractional differential equations with uncertainty," Nonlinear Analysis: Theory, Methods \& Applications, vol. 74, no. 11, pp. 3685-3693, 2011.

[2] A. Harir, S. Melliani, and L. S. Chadli, "Existence and uniqueness of a fuzzy solution for some fuzzy neutral partial integro-differential equation with nonlocal conditions," Journal of General Letters in Mathematics, vol. 5, no. 1, pp. 7-14, 2018.

[3] A. Harir, S. Melliani, and L. S. Chadli, "Existence and uniqueness of a fuzzy solution for some fuzzy neutral partial differential equation with nonlocal condition," International Journal of Mathematics Trends and Technology, vol. 65, no. 2, 2019.

[4] A. Harir, S. Melliani, and L. S. Chadli, "Fuzzy space-time fractional telegraph equations," International Journal of Mathematics Trends and Technology, vol. 64, no. 2, pp. 101108, 2018

[5] S. Salahshour, T. Allahviranloo, and S. Abbasbandy, "Solving fuzzy fractional differential equations by fuzzy Laplace transforms," Communications in Nonlinear Science and Numerical Simulation, vol. 17, no. 3, pp. 1372-1381, 2012.

[6] C. G. Gal and S. G. Gal, "Semigroups of operators on spaces of fuzzy-number-valued functions with applications to fuzzy differential equations," 2013, http://arxiv.org/abs/1306.3928v1.

[7] P. Diamond and P. E. Kloeden, Metric Spaces of Fuzzy Sets: Theory and Applications, World Scientific, Singapore, 1994.

[8] K. B. Oldham and J. Spanier, The Fractional Calculus, Academic Press, New York, NY, USA, 1974.

[9] H. Y. Goo and J. S. Park, "On the continuity of the Zadeh extensions," Chungcheong Mathematical Society, vol. 20, no. 4, pp. 525-533, 2007.

[10] M. L. Puri and D. A. Ralescu, "Differentials of fuzzy functions," Journal of Mathematical Analysis and Applications, vol. 91, no. 2, pp. 552-558, 1983.

[11] S. Seikkala, "On the fuzzy initial value problem," Fuzzy Sets and Systems, vol. 24, no. 3, pp. 319-330, 1987.

[12] N. V. Hoa, "Fuzzy fractional functional integral and differential equations," Fuzzy Sets and Systems, vol. 280, pp. 58-90, 2015.

[13] O. Kaleva, "The Cauchy problem for fuzzy differential equations," Fuzzy Sets and Systems, vol. 35, no. 3, pp. 366-389, 1990.

[14] O. Kaleva, "Fuzzy differential equations," Fuzzy Sets and Systems, vol. 24, no. 3, pp. 301-317, 1987.

[15] N. V. Hoa, "Fuzzy fractional functional differential equations under Caputo gH-differentiability," Communications in 
Nonlinear Science and Numerical Simulation, vol. 22, no. 1-3, pp. 1134-1157, 2015.

[16] S. Salahshour, A. Ahmadian, S. Abbasbandy, and D. Baleanu, " $M$-fractional derivative under interval uncertainty: theory, properties and applications," Chaos, Solitons and Fractals, vol. 117, pp. 84-93, 2018.

[17] H. Brezis, Opérateurs Maximaux Monotones et Semi-groupes de Contractions dans les Espaces de Hilbert, Math. Studies 5, North-Holland, Amsterdam, Netherlands, 1973.

[18] T. Allahviranloo and M. B. Ahmadi, "Fuzzy Laplace transforms," Soft Computing, vol. 14, no. 3, pp. 235-243, 2010.

[19] E. Bazhlekova, Fractional evolution equations in Banach spaces, Ph.D. thesis, Eindhoven University of Technology, Eindhoven, Netherlands, 2001.

[20] A. Pazy, Semigroups of Linear Operators and Applications to Partial Differential Equations, Applied Mathematical Sciences, Vol. 44, Springer, New York, NY, USA, 1983. 


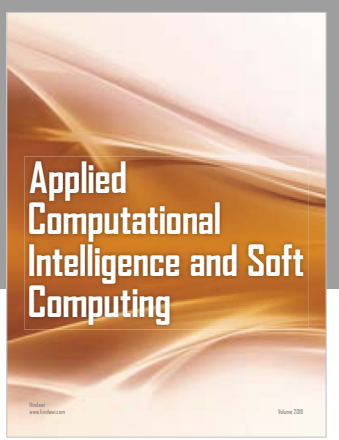

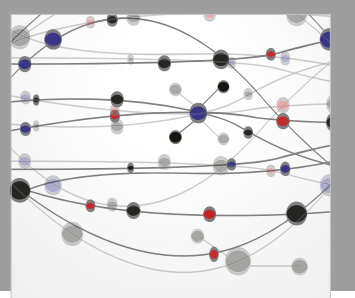

The Scientific World Journal
Submit your manuscripts at

Computing
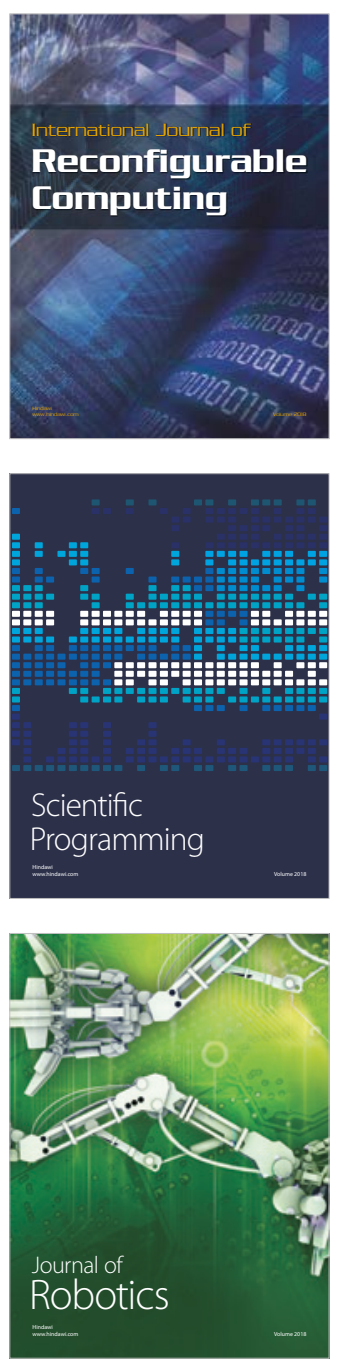

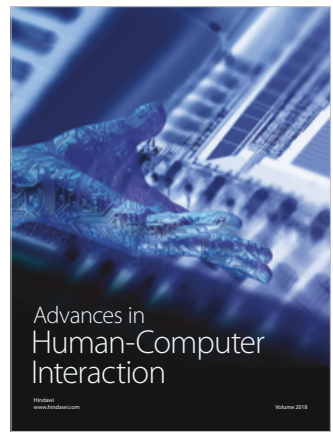

Human-Compute

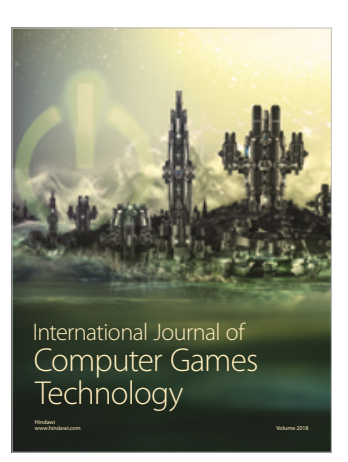

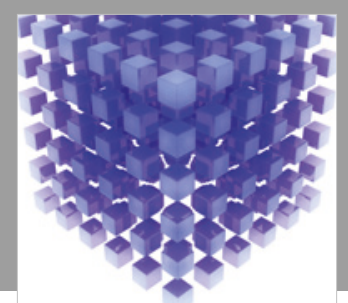

Mathematical Problems in Engineering

\section{Engincering}
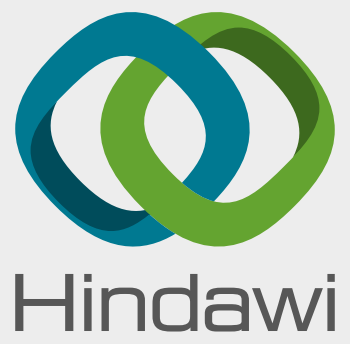

www.hindawi.com
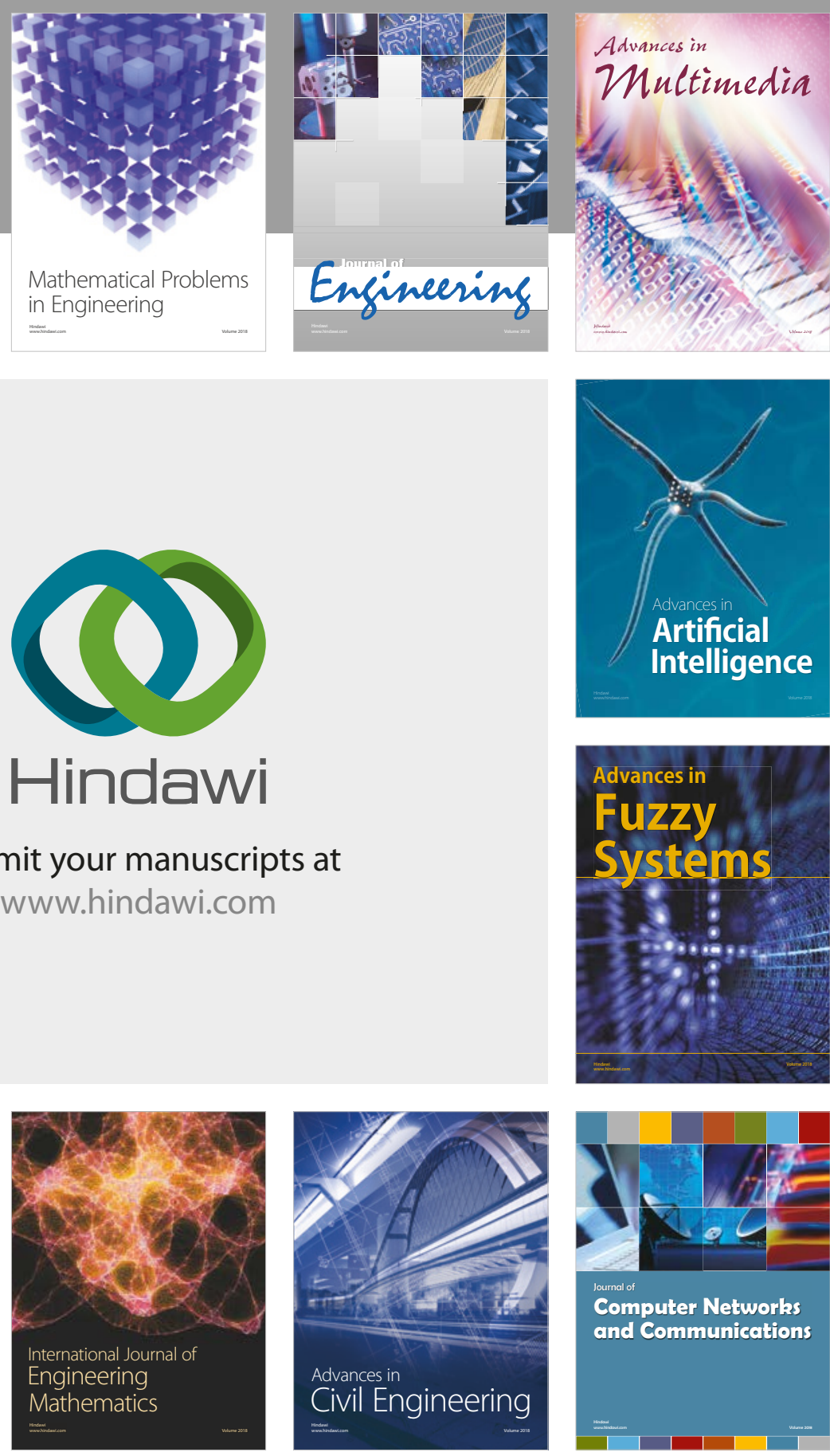

Computer Networks and Communications

Multimedia
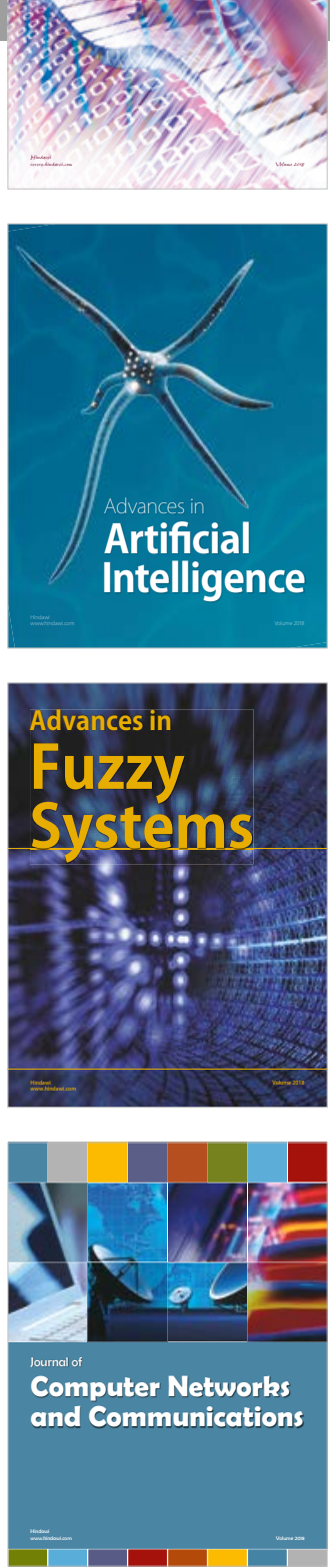

Advances in

Modelling \&

Simulation

in Engineering

interaction

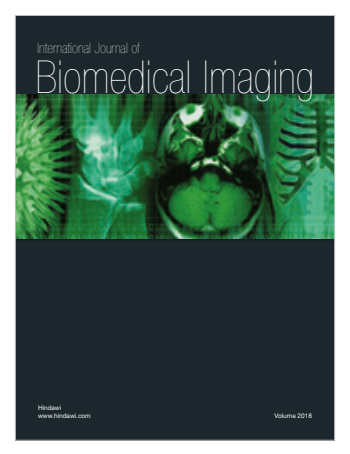

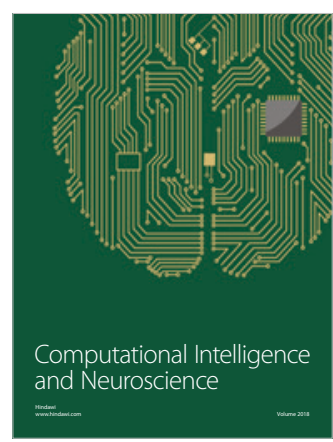

